\title{
CONSIDERACIONES PARA UNA TEORÍA LINGÜÍSTICO-INTERDISCIPLINARIA DEL TEXTO JURÍDICO COMO FUNDAMENTO DE UNA CIENCIA DEL DERECHO
}

\author{
PEDRO AULLÓN DE HARO \\ (Universidad de Alicante)
}

0 . Es sin duda fenómeno curioso el hecho de la señaladísima parquedad bibliográfica que todavía existe en torno al estudio, bajo presupuestos científico-humanísticos actuales, del texto jurídico en cuanto realidad lingüistica susceptible de disfrutar muy rentablemente de los refinados procedimientos de gran altura tecnológica del más moderno análisis lingüístico-textual junto a la asunción integradora del pensamiento y del extraordinario aparato de la Retórica clásica, según viene a confirmar el último estadio decisorio de este tipo de investigaciones ${ }^{1}$. Asimismo se nos aparece como francamente urgente la asimilación globalizadora de dichos puntos de vista disciplinarios en el marco de una epistemología de principio estructural capaz de poner en marcha, desde tales criterios, un orden y técnica descriptivos que, aprovechando inteligentemente la tradición teórico-jurídica más utilizable a este propósito, disponga orgánicamente los fundamentos de una Ciencia del Derecho sobre bases lingüístico-interdisciplinarias 0 , si se prefiere, sencillamente interdisciplinarias ${ }^{2}$.

1 Vid., en este sentido, las muy importantes aportaciones realizadas por el profesor Garcia Berrio en torno a este tema y recogidas en Garcia Berrio, 1984a y 1984b.

2 El papel de primera línea desempeñado por la lingüistica contemporánea dentro de la serie de las Ciencias Humanas, en virtud de la prodigiosa extensión de su campo de actividades y de la evidente conexión del mismo con otras disciplinas susceptibles de recibir una gran experiencia acumulada por medio de realidades descritas o explicadas o, en otro sentido, metodológicamente utilizables por su carácter ejemplar y éxito científico, ha dado lugar, sin embargo, a los comienzos de una autocritica en última instancia sustentada por los grandes progresos de la propia disciplina. Se ha podido recientemente hablar, asi, por ejemplo, de crisis metodológica de superproducción (Garcia Berrio, 1984a: 18-19, 50, 1984b), o, dicho con otras palabras, puesto que las materias de fundamento lingüístico han venido ya a realizar complejísimas operaciones sobre los lenguajes estándar y literario mediante potentes modelos teórico-analíticos, una vez llegados tan lejos no parece sino que haya que volver la vista atrás, sobre todo cuando los mismos niveles alcanzados presuponen en ciertas ocasiones tal grado de complicación que quizás ya sea muy difícil saber para qué pudieran servir. Naturalmente, esto no quiere decir que no quede aún mucho por hacer incluso dentro de esos mismos estadios. Existe, además, otro tipo de autocrítica, esta vez de rasgos especialmente ideológicos, sustentada recientemente por quienes piensan, casi en términos globales humanos, que las grandes tecnologias lingüísticas 
Parece inevitable la gran dificultad que existe a la hora de abordar interdisciplinariamente un objeto de estudio cualquiera. La extensión complejísima de los desarrollos teóricos y científicos actuales comprensiblemente hace sentir impotencia al estudioso, por lo común incapaz de sobreponerse a un devenir vertiginoso de multiplicadas y superpuestas descripciones de las series determinables de la realidad, por no hablar ya de sus totalizaciones - aun provisionalmente-posibles. Todo conviene en desbordar los limitados años de vida intelectual de un individuo, si es que la vida, por sí misma, no lo deja en el camino. La flexibilidad, lo acertado de las estrategias de trabajo, el antidogmatismo teórico-científico y la formación de equipos de estudio interdisciplinario se presentan, en esta situación, como la única alternativa a tal estado de cosas. De principio, a partir de ahí, pensamos que ya cabe abrigar importantes y fundadas expectativas.

El presente artículo consiste simplemente en la exposición de algunas consideraciones fundamentales en torno a una propuesta interdisciplinaria posible destinada al estudio del texto juridico como fundamento de una Ciencia del Derecho sujeta a todos los requisitos científico-humanísticos actuales. En el caso de rentabilizarse debidamente, o con éxito tan sólo en alguna de sus parcelas, estamos convencidos de su

constituyen al fin un apoyo de las Ciencias Humanas al propio desastre de nuestra civilización tecnológica. Se trataría, pues, de volver a la exégesis, a la vieja tradición europea empezando a olvidar una barbarie. Ambos tipos de autocrítica han sido presentados por autores españoles: A. Garcia Berrio en el primer caso y M. Crespillo (1986) en el segundo. No es éste el momento de efectuar una critica de las mismas, aunque hemos de confesar que de buen grado nos sentimos de acuerdo con la mayor parte de sus argumentos, pero si de advertir acerca de la peligrosidad de muy distinto orden que encierran. En cualquier caso, lo dicho y la multitud de supuestos que contienen nos servirán para decir, con toda sencillez, que tales autocríticas carecen totalmente de validez para quienes no se hallen en situación científica análoga a la de quienes las han formulado. La particular historia y el presente de cada disciplina con frecuencia determinan realidades científicamente muy alejadas y, aparte de toda discusión de índole epistemológica interna o bien de referencialidad externa, el hecho es que conviene tener claro que no se puede estar de vuelta sin haber ido. Distinta cuestión es que no interese o no se pretenda ir aquí o allá. Pero, de todos modos, volviendo a nuestra razón lingüística, el dato positivo incuestionable consiste en que las ciencias del lenguaje han venido a representar una modelización científicohumanistica altísimamente productiva para la mayor parte de las Ciencias Humanas, desde la antropología a la psicología. Por ejemplo, el sociólogo R. Boudon argüia, en 1974, cómo la gran progresión de las modernas ciencias del lenguaje había tenido lugar a partir del momento en que el lingüista decidió aplicarse a la delimitación de problemas aislados y abstractos como es el de la determinación de las unidades mínimas fonológicas y, desde ahí, al enfrentarse a estructuras mayores o de otro nivel, procedió a construir modelos formales capaces de engendrar las propiedades estructurales de las lenguas. Así es como se interroga Boudon por qué razón la lingüística o la economía han pasado de manera relativamente brutal de un estado de polimorfismo notorio a una tradición de investigación lineal (Boudon, 1974: 14, 32). Desde otra disciplina bien distante de la sociologia, C. Castilla del Pino ha emprendido la aplicación lingüistico-formal al texto literario (Castilla del Pino, 1984) y, asimismo, ha podido decir en otro lugar: "[...] trato de hacer ver que la Psico(pato)logia tiene que discurrir por los caminos de esa ciencia, hoy en notable desarrolio y de resultados admirables, que es la Lingüistica. No hay razón alguna para que muchos de los análisis que se verifican en el ámbito de la lengua y el habla normales no se apliquen al habla de psicóticos, neuróticos, etc. (Castilla del Pino, 1984b: 10). No se piense, ni mucho menos, que intentamos hacer entender que en la lingüística existe un completo arsenal de soluciones para todos los problemas científico-humanísticos, sino poner de manifiesto lo más sucintamente posible el estado de la cuestión. Por lo demás, la ya extensa historia de la formalización y de la matematización de las Ciencias humanas hace cada vez más necesaria la realización de su crónica y su evaluación pertinente. Por último, no queremos dejar de señalar cómo el propósito de la teoria del texto jurídico que en estas páginas consideramos se incardina en último término en un propósito mucho más amplio y que centralmente se expuso desde el ángulo de la Filología general. Contribución a una Teoría de las Ciencias Humanas" (Aullón de Haro. 1984b). 
inocultable proyección o capacidad de amplificación científica en el campo de las diversas actividades disciplinarias jurídicas, ya sean teóricas o incluso prácticas.

1. En primer término adelantaremos la idea de que, a nuestro juicio, el eje principal de una Ciencia del Derecho ha de basarse sustancialmente en el análisis, junto a la necesaria formulación teórico-prescriptiva y a los posteriores estudios de las consecuencias operadas sobre la realidad —extratextual-, del texto jurídico. Si la constitución ontológica del texto jurídico, como la de cualquier otro texto de lengua natural, corresponde obviamente a determinados mecanismos de producción lingüísticos, es evidente que el objeto de estudio que se nos impone viene necesariamente a coincidir, en principio, con el objeto mismo de la Filología, aunque desde una perspectiva semántica juridica. La ostensible diferencia que en este sentido existe entre Ciencia del Derecho y Filologia consiste, únicamente, en que para la primera de ellas se trata exclusivamente de abordar, al margen de la más estricta o neutra inmanencia, una específica tipología, la correspondiente al texto jurídico, dentro de las tipologías globales de la producción lingüística general.

Será, por tanto, procedente comenzar por discernir nuestro objeto de estudio en el cuadro tipológico general de los lenguajes naturales, puesto que si bien dicho objeto no poseerá, desde criterios puramente jurídicos, una finalidad operacional última lingüística, sí será, sin embargo, un imprescindible instrumento encaminado a obtener los fundamentos teóricos y las descripciones necesarias para la constitución de una Ciencia del Derecho actualizadamente asentada sobre las mejores disponibilidades tanto epistemológicas como metodológicas que las disciplinas humanísticas hoy ofrecen.

Han sabido ver distintos autores que las producciones lingüístico-jurídicas son determinables en el marco de tres grandes vertientes: 1) la de los textos normativos o de ley; 2) la de los textos producidos por las actividades de expresión lingüística ejercidas en las diversas instancias judiciales, de uso institucional, etc., y 3 ) la de los textos que versan sobre los referidos textos de función y de naturaleza 1 y $2^{3}$. Sobre este esquema de delimitación de los lenguajes jurídicos se advertirá que, dentro del sistema global de los distintos tipos de producción lingüística —lenguaje estándar, lenguaje literario y lenguaje científico-, las anteriores vertientes 1 y 2 son adscribi-

3 Cfr., en lengua española, el excelente y culturalmente bien formado libro de principios hartmannianos de S. Soler (Soler, 1969), además del de G. R. Carrió (Carrió, 1965), trabajo que en buena parte podriamos denominar pragmático-lingüistico. Por su parte, escribe Soler: «Piénsese en el equivoco que resultaria para la Química o la Botánica si en ellas se reprodujera la misma confusión entre la ciencia y el objeto de que ellas se ocupan. La Botánica (ciencia) se ocupa de los vegetales (objeto); la Química (ciencia) se ocupa de la molécula (objeto). Pues bien: el Derecho (ciencia) se ocupa del derecho (objeto). Para mayor pena todavía, debe observarse que si bien la Botánica y la Química se componen de palabras y símbolos y que con esos medios operan y se expresan, sus objetos, en cambio, son cosas reales consistentes. La ciencia del Derecho también se compone, desde luego, de enunciados verbales, en lo cual no se aparta mucho de otras ciencias; pero la particular dificultad consiste en que en ella el objeto de que se ocupa, a diferencia de las otras ciencias, consiste también en palabras, en enunciados verbales, en proposiciones" (Soler, 1969: 139-140). Ahora bien, lo que Soler entiende por una pena y una particular dificultad no es sino el hecho de que se trata de objetos de naturaleza distinta, y a tal realidad corresponderán procedimientos de naturaleza y función diversa. Más grave aún seria, en ese sentido, el problema de la lingüística, en la cual ciencia y objeto como fragmentos de una misma realidad de lengua se resuelven en términos de funcionalidad metalingüistica a efectos disciplinarios. 
bles, en último término, a un subapartado del lenguaje estándar, aun con todos los distingos y especificidades - tipo de circuito comunicativo, alto índice de frecuencia de clichés, acrónimos, etc.-que acerca de las mismas cupiera efectuar. Por contra, la vertiente determinada por el enunciado 3 , es decir, la correspondiente a aquellos textos que versan sobre textos normativos o de ley y sobre textos dependientes de las restantes actividades juridicas de expresión lingüística, es clasificable, sin ningún género de dudas y con todas las precisiones que se quiera hacer, en el apartado relativo al lenguaje científico dentro del sistema global de los distintos tipos de producción lingüística. Este caso consiste, ciertamente, en textos cuyo discurso es de naturaleza teórica o metateórica y de función científica o metacientífica, o, lo que es lo mismo, en textos compuestos de discursos metalingüísticos aplicados a textos de lengua-objeto, bien de constitución ética (Pike, 1967: 37-38; Dressler, 1974: 24-25), bien de consideración émica (Pike, 1967: 37-38; Dressler, 1974: 24-25). Por su parte, ni el texto jurídico normativo ni el texto jurídico de emisión institucional, pongamos por caso, describen discursos de tipo científico, aunque bien es verdad que su expresión lingüística siempre deberá estar regida por el mayor grado factible de funcionalidad denotativa, siendo técnicamente más perfectos en la medida en que así suceda.

2. Una Ciencia del Derecho esencialmente concebida como estudio de los textos normativos o de ley y de los textos dependientes de las restantes actividades jurídicas de expresión lingüística presupone, naturalmente, un eje de análisis sincrónico y otro diacrónico, ciertamente dentro del cuadro general socio-histórico y de la estructura pragmática de los circuitos comunicativos y culturales que éste subsume. Asimismo, cometido irrenunciable de la Ciencia del Derecho ha de ser la observación disciplinaria, según tradicionalmente preconizó el realismo jurídico, de los hechos de realidad provocados por las diversas aplicaciones de la norma jurídica y, en otro sentido, de los fenómenos de realidad económico-política y socio-cultural que propiciaron la enunciación de la norma, pero siempre desde el punto de mira sistemático de una teoría pragmática ${ }^{4}$.

4 No se trata ahora, esperamos que se entienda, de sostener una postura doctrinal realista frente a su habitual opuesta representada por el dogmatismo. Nos vamos a permitir, por otra parte, traer a colación en extenso un interesante fragmento de $M$. Foucault, cuya lectura, sin más comentarios, hará obtener múltiples y valiosas conclusiones a éste y otros propósitos. Describe Foucault, aludiendo al litigio entre individuos en el derecho feudal: «En primer lugar habia pruebas sociales, pruebas de la importancia social de un individuo. En el viejo derecho de Borgoña del siglo $\mathrm{Xl}$, el acusado de asesinato podía establecer perfectamente su inocencia reuniendo a su vez doce testigos que juraban que él no habia cometido asesinato alguno. El juramento, por ejemplo, no se fundaba en haber visto con vida a la presunta victima o en una coartada para el presunto asesino. Para prestar juramento, atestiguando que un individuo no había matado, era necesario ser pariente del acusado [...]. En segundo lugar, había pruebas de tipo verbal. Cuando un individuo era acusado de algo - robo o asesinato- debia responder a esta acusación con cierto número de fórmulas, garantizando que no había cometido delito. Podia suceder que el individuo fracasara o tuviera éxito al pronunciar estas fórmulas. En algunos casos se pronunciaba la fórmula y se perdía, no por haber mentido o por haberse probado que se había mentido, sino simplemente porque no se había pronunciado la fórmula correctamente. Un error de gramática, un cambio de palabras invalidaba la fórmula y no la verdad de lo que se pretendía probar. Es evidente que en el nivel de la prueba sólo se trataba de un juego verbal porque en el caso de un menor, una mujer o un padre, el acusado podía ser sustituido por otra persona. Esta otra persona, que, tiempo después en la historia del derecho se convertiria en el abogado, era quien debia pronunciar las fórmulas en lugar del acusado. Si se equivocaba al pronunciarlas, aquél a quien reemplazaba perdía el proceso" (Foucault, 1983: 69-70). 
En realidad, el gran peligro de desajuste disciplinario radica, a nuestro juicio, en que el espesor y la multiplicidad del deseable carácter totalizador de la edificación que se propone consiga en determinados tramos hacer perder de vista el sentido estructural completo describible a partir del núcleo concreto del objeto de estudio: el texto jurídico. Por lo demás, creemos fácilmente entendible el hecho de dar por sentado que la construcción del modelo lingüístico-teórico inmanente del texto-objeto jurídico representa en todo caso el previo paso estructural necesario para la objetivación de su trascendencia, finalmente jurídico-pluridisciplinaria.

La interpretación de la normativa juridica ha supuesto, desde luego, un sector amplísimo y variadísimo de los estudios de Derecho ${ }^{5}$. Sin embargo, pese a poder ser comprendida dicha normativa como sistema de proposiciones, puede decirse que hoy por hoy los análisis habitualmente practicados, incluso los de mayor competencia y hasta los de proyecto lógico-lingüístico más claro ${ }^{6}$, se hallan muy lejos de un posible acceso a los modelos de la ciencia lingüística actual. Por ejemplo, la relativamente frecuente consideración especulativa, además de débilmente sistemática, del carácter pragmático-axiológico de las estructuras de relación ideología/norma jurídica o de algún elemento relevante de su funcionamiento, como es el de los estereotipos lingüisticos ${ }^{7}$, no pasa de ser, según creemos, la acumulación más o menos atomista de problemas cuyo tratamiento específico carece de auténtica fundamentación entrevisto fuera de un modelo descriptivo, es decir, en situación ajena a un dispositivo estructurado de pies a cabeza por una disciplina científica o por un proyecto teórico que ha de evolucionar según los desarrollos particulares urgidos por la imposición de las diferentes perspectivas de la realidad. Sea como fuere, es obvio que no puede trazarse un estudio del sistema del lenguaje jurídico sobre la base central de un mero rasgo o componente, por mucha intencionalidad o influencia extralingüística que pudiera ejercer la instalación del mismo en el seno del discurso.

Parece claro, por otra parte, que el proceder a inventariar lexicones, catálogos de estereotipos o alguna clase posible de repertorio jurídico mediante principios retóricos y lingüísticos dentro de una teoría integrada de rango textual haría cambiar en mucho la imagen y las dimensiones del conocimiento propiamente jurídico ${ }^{8}$.

5 Para el lector no jurista probablemente sería ilustrativo componer un breve muestreo de disparidades interpretativas. Vid., a este respecto, Danz, 1955; Cesari, 1963; Cueto Rua, 1981 y Ollero, 1982.

6 En España se ha de tener muy en cuenta el importante trabajo de J. R. Capella (Capella, 1968). Se trata de un Jibro que, dentro de los márgenes del positivismo lógico, sigue a von Wright (Norm and Action), a Kalinowski (Introduction à la logique juridique), a Quine (Sentido y métodos) y a Sacristán (Introducción a la lógica simbólica y al análisis formal) y no contiene, por lo demás, apenas ningún aprovechamiento de teoría ingüistica. Sólo el capítulo $V$ estudia los conceptos, que nosotros calificariamos de función pragmática, de Edictor, Sujeto y Sanción en la norma jurídica (Capella, 1968: 147-189), y el capítulo VII está dedicado al lenguaje legal y al lenguaje natural (Capella, 1968: 242-275).

7 Puede verse, en este sentido, el no descaminado estudio de carácter antidogmático, aplicado al fenómeno lingüístico-ideológico de los estereotipos, de L. A. Warat (Warat, 1973), en su tomito en colaboración con A. Anselmo Martino (Anselmo Martino, 1973). Vid., igualmente, entre algún otro posible, Núñez Ladeveze, 1980.

8 En general, de entre los volúmenes dedicados al estudio del lenguaje del Derecho -aparte de otras destacadas referencias que haremos después al aludir a la ciencia retórica- acaso sea la edición de U. Scarpelli titulada Diritto e analisis del linguaggio (Scarpelli, 1976) lo más valioso que conocemos. Está integrada por artículos predominantemente "positivistas», y, por supuesto, de principios lingüísticos cuando menos pre-textuales, de los que convendrá dejar constancia: J. L. Austin («Enunciati performativi"), A. Ross ( TTû-tû»), N. Bobbio ( Scienza del diritto e analisi del linguaggio»), J. Wróblewsky («Una base semantica per la teoria dell'interpretazione giuridica») y G. R. Carrió («l giudici creano diritto»). También debe recordarse, a este 
3. La propuesta que aquí se expone en cuanto modelo teórico nuclear para una Ciencia del Derecho consiste, tomando como puntos de referencia inexcusables los importantes presupuestos de Antonio García Berrio para la elaboración de una Retórica general, entendida como ciencia general del discurso, y de una Retórica general literaria, considerada como ciencia general del discurso específicamente literario (García Berrio, 1984a, 1984b), en la instrumentalización de una Retórica general jurídica integradora de las ciencias clásicas -Poética y Retórica- y de las ciencias modernas del discurso - lingüistica del texto y Poética lingüística, principalmente— 9 . La planificación de una Retórica general jurídica asimiladora del moderno instrumental analítico lingüístico-textual al proceso del fecundísimo esquema clásico constituido por las operaciones retóricas fundamentales de inventio, dispositio y elocutio permite entrever la construcción totalizadora de un sistema que arrojaría luz decisiva y habría de constituir vertebralmente por sí mismo un auténtico edificio científico-jurídico.

No se puede olvidar aquí la importante contribución de Theodor Viehweg a una teoría de la tópica jurídica dirigida hacia un modelo técnico de práctica jurídica (Viehweg, 1964) ${ }^{10}$, pero tampoco omitir el hecho de que la delimitación de una estructura tópica no deja de ser, pese a su extraordinaria relevancia, la concreción de una rama de entre las que componen la totalidad de un árbol. Igualmente no podemos dejar de mencionar los estudios de Charles Perelman y otros acerca de la argumentación y la retórica del Derecho ${ }^{11}$.

En realidad, la determinación textual jerarquizadora, resoluble en sistemas de estructuras consteladamente vinculadas, del volumen de topoi jurídicos históricamente existente ${ }^{12}$, permitiría reconstruir el sistema sincrónico y la evolución de los

propósito, la obra de S. Pugliatti titulada Grammatica e diritto (Pugliatti, 1978), además de la no muy rigurosa miscelánea contenida en E/ discurso juridico (Pugliatti, 1982). No centrado en problemas de lenguaje, pero sien cuestiones histórico-documentales que le atañen, es el trabajo breve y preciso de A. Montoya Melgar (Montoya Melgar, 1975).

9 El paso de la oración al texto como fundamental objeto de estudio ha constituido indudablemente una poderosa progresión en los ámbitos de la ciencia lingüistica y de todas aquellas disciplinas con ésta relacionada. En lo que más concretamente aqui nos compete, importa referir de manera muy especial la alusión de T. A. van Dijk a la jurisprudencia, junto a otras materias, como posible objeto lingüístico-textual (van Dijk, 1983), así como los estudios de R. Leodolter (Leodolter, 1975) y de D. Rave (Rave (ed.), 1971).

10 El libro de Th. Viehweg Tópica y jurisprudencia (Viehweg, 1964) es obra de brillante envergadura, si bien el paso de más de veinte años ha puesto especialmente en evidencia su aislamiento tanto en materia retórica como, sobre todo, en materia lingüistica. La traducción española va precedida de un prólogo del profesor García de Enterria, quien ha sabido ver con gran entusiasmo la relevancia de la obra.

11 Cfr. Perelman, 1970, autor que, ya en 1958, abordó, junto a L. Olbrechts-Tyteca, un tratado de la argumentación (Perelman-Olbrechts-Tyteca, 1958). Una bibliografía esencial sobre el estado actual de la discusión en torno a la Retórica puede ser la siguiente: Barilli, 1979; Breuer, 1974; Cerisola, 1983; ChristensenChristensen, 1978; van Dijk, 1972; van Kijk, 1983; Florescu, 1971; Garcia Berrio, 1984a, 1984b; Garcia Berrio-Albaladejo, 1983; Heilmann, 1983b; Kibedi Varga, 1970; Kopperschmidt, 1973; Leoni Pigliasco (eds.), 1979; López; 1985; Martin, 1974; Mosconi y otros, 1982; Plett (ed.), 1977; Pozuelo, 1986, 1987; Rehbock, 1980; Richards, 1965; Ritter-Santini-Raimondi (eds.), 1978; Spillner, 1979 y Valesio, 1980.

12 A propósito de este tema, de obligada mención y consideración es la importantísima Tópica de Cicerón, de naturaleza fundamentalmente jurídica (Cicerón, 1902-1922). Téngase en cuenta, además, en relación con ello, la existencia de, al menos, dos importantes trabajos de recopilación y de explicación léxico-jurídica en nuestro pais, el Diccionario de castellano jurídico de J. Delgado Garzón (Delgado Garzón, 1987) y el Diccionario de Derecho de L. Ribó (Ribó, 1987). 
sistemas del pensamiento jurídico hasta alcanzar la actualidad. Solamente a partir de todo ello cabe pensar, por otra parte, retomando, quizás, el pensamiento de Wilhelm Dilthey (Dilthey, 1980: 105 ss.), en la posibilidad real de la conjunción lícita de la Ciencia del Derecho dentro de la serie, según su estado actual, de las Ciencias Humanas.

\section{Referencias bibliográficas}

Althaus, H. P.; Henne, H. y Wiegand, H. E. (eds.) (1980), Lexikon der germanistischen Linguistik, 2, Tubinga, Max Niemeyer.

Anselmo Martino, A. (1973), Lenguaje y definición jurídica, Buenos Aires, Cooperadora de Derecho y Ciencias Sociales.

Aullón de Haro, Pedro (coord.) (1984a), Introducción a la Crítica literaria actual, Madrid, Playor.

- (1984b), «Por una Filología general. Contribución a una Teoría de las Ciencias humanas», Málaga, Universidad de Málaga.

Barilli, R. (1979), La retorica, Milán, Isedi.

Boudon, Raymond (1974), La crisis de la Sociología, Barcelona, Laia.

Breuer, D. (1974), Einführung in die pragmatische Textheorie, Munich, Wilhelm Fink. Capella, J. R. (1968), El Derecho como lenguaje. Un análisis lógico, Barcelona, Ariel. Carrió Genaro, R. (1965), Derecho y lenguaje, Buenos Aires, Abeledo-Perrot. Castilla del Pino, Carlos (1984a), Teoria de la alucinación, Madrid, Alianza.

- (1984b), «El psicoanálisis y e! universo literario», en Aullón de Haro, Pedro (coord.) (1984a), pp. 251-345.

Cerisola, P. L. (1983), Trattato di retorica e semiotica Letteraria, Brescia, La Scuola. Cesari, A. (1963), L'interpretazione dei contratti collectivi, Milán, Giuffré.

Cicerón (1902-1922), Obras completas, traducción por M. Menéndez Pelayo, Sucesores de Hernando.

Crespillo, M. (1986), Historia y mito de la lingüistica transformativa. Madrid, Taurus. Cueto Rua, J. C. (1981), Judicial Methods of Interpretation of the Law, Louisiana, State University.

Christensen, F.; Christensen, B. (1978), Notes Toward a New Rhetoric. Nine Essays for Teachers, Nueva york, Harper \& Row.

Danz, E. (1955), La interpretación de los negocios juridicos, Madrid, Revista de Derecho Privado.

Delgado Garzón, J. (1987), Diccionario de castellano jurídico, Madrid, Pons.

Díez Borque, J. M. (ed.) (1985), Métodos de estudio de la obra literaria, Madrid, Taurus.

Dijk, T. A. van (1972), Some Aspects of Text Grammars. A Study in Theoretical Linguistics and Poetics, La Haya-París, Mouton.

- (1983), La ciencia del texto. Un enfoque interdisciplinario, Barcelona, Paidós.

Dilthey, W. (1980), Introducción a las Ciencias del Espiritu, Madrid, Alianza.

Dressler, W. U. (1974), Introduzione alla linguistica del testo, Roma, Officina

Florescu, V. (1971), La Retorica nel suo sviluppo storico, Bolonia, II Mulino.

Foucault, Michel (1983), La verdad y las formas juridicas, México, Gedisa.

García Berrio, A. (1984a), «Más allá de los 'ismos': Sobre la imprescindible globalidad crítica», en Aullón de Haro, P. (coord.) (1984a), pp. 347-387. 
- (1984b), «Retórica como ciencia de la expresividad. (Presupuestos para una Retórica general.)", en Estudios de IIngüistica, 2, pp. 7-59.

García Berrio, A.; Albaladejo, T. (1983), «Estructura composicional. Macroestructuras», en Estudios de Lingüistica, 1, pp. 127-180.

Heilmann, L. (1983a), Linguaggio, Lingue, Culture. Saggi linguistici e indologici, Bolonia, II Mulino.

- (1983b), «Rhetoric, New Rhetoric and Linguistic Theory», en Heilmann, L. (1983a), pp. 283-299.

Kibedi Varga, A. (1970), Rhétorique et littérature. Etudes de structures classiques, París, Didier.

Kopperschmidt, J. (1973), Allgemeine Rhetorik. Einführung in die Theorie der persuasiven Kommunikation, Stuttgart-Berlín-Colonia-Maguncia, W. Kohlhammer.

Leodolter, R. (1975), Das Sprachverhalten von Angeklagten bei Gericht, Kronberg, Scriptor.

Leoni, F. A.; Pigliasco, M. R. (eds.) (1979), Retorica e scienze del linguaggio. Atti del X Congresso Internazionale di Studi, Pisa, 31 maggio-2 giugno 1976, Roma, Bulzoni.

López, Á. (1985), «Retórica y lingüística: una fundamentación lingüística del sistema retórico tradicional», en Díez Borque, J. M. (ed.) (1985), pp. 601-653.

Martin, J. (1974), Antike Rhetorik. Technik und Methode, Munich, C. H. Beck'sche Verlagsbuchhandlung.

Montoya Melgar, A. (1975), Ideologia y lenguaje en las primeras leyes laborales de España, Madrid, Civitas.

Mosconi, G. y otros (1981), Discorso e Retorica, Turín, Einaudi.

Núñez Ladeveze, L. (1980), Lenguaje juridico y ciencia social, Madrid, Akal.

Ollero, A. (1982), Interpretación del Derecho y positivismo legalista, Madrid, Edersa.

Perelman, Ch. (1970), La lógica jurídica y la nueva retórica, Madrid, Civitas.

Perelman, Ch.; Olbrechts-Tyteca, L. (1958), La Nouvelle Rhétorique. Traité de l'Argumentation, París, P.U.F.

Pike, K. L. (1967), Language in Relation to a Unified Theory of the Structure of Human Behavior, La Haya, Mouton.

Plett, H. F. (ed.) (1977), Rhetorik, Kristische Positionen zum Stand der Forschung, Munich, Wilhelm Fink.

Pozuelo, J. M. (1986), «Retórica y narrativa: la narratio», en Epos, II, pp. 231-252.

- (1987), «Neorretórica y Retórica general», en Dispositio (en prensa).

Pugliatti, S. (1978), Grammatica e diritto, Milán, Giuffré.

- (1982), El discurso juridico, Buenos Aires, Hachette.

Rave, D. (1971), Paraphrasen juristicher Texte, Darmstadt, Interdisziplinäre Arbeitsgruppe «Analyse der juristischen Sprache».

Rehbock, H. (1980), «Rhetorik», en Althaus, H. P. - Henne, H.-Wiegand, H. E. (eds.) (1980), pp. 293-303.

Ribó, L. (1987), Diccionario de Derecho, Barcelona, Bosch.

Richards, I. A. (1965), The Philosophy of Rhetoric, Nueva York, Oxford University Press.

Ritter-Santini, L.; Raimondi, E. (eds.) (1978), Retorica e critica letteraria, Bolonia, II Mulino.

Scarpelli, U. (1976), Diritto e analisi del linguaggio, Milán, Edizioni di Comunità. Soler, Sebastián (1969), Las palabras de la ley, México, F.C.E. 
Spillner, B. (1979), Lingüistica y literatura. Investigación del estilo, retórica, lingüística del texto, Madrid, Gredos.

Valesio, P. (1980), Novantiqua. Rhetorics as a Contemporary Theory, Bloomington, Indiana University Press.

Viehweg, Th. (1964), Tópica y jurisprudencia, Madrid, Taurus.

Warat, L. A. (1973), «La lingüistica jurídica, la problemática definitoria y el condicionamiento ideológico del accionar humano", en Anselmo Martino, A. (1973), pp. 17-58. 